\title{
Baculovirus-based genome editing in primary cells
}

\author{
Maysam Mansouri ${ }^{\mathrm{a}, \mathrm{b}}$, Zahra Ehsaei ${ }^{\mathrm{c}}$, Verdon Taylor ${ }^{\mathrm{c}}$, Philipp Berger ${ }^{\mathrm{a}, *}$ \\ a Paul Scherrer Institute, Biomolecular Research, Molecular Cell Biology, CH-5232 Villigen, Switzerland \\ ${ }^{\mathrm{b}}$ ETH Zürich, Department of Biology, $\mathrm{CH}-8093$ Zürich, Switzerland \\ c University of Basel, Department of Biomedicine, $\mathrm{CH}-4058$ Basel, Switzerland
}

\section{A R T I C L E I N F O}

\section{Article history:}

Received 18 November 2016

Received in revised form 19 January 2017

Accepted 19 January 2017

Available online 22 January 2017

\section{Keywords:}

Genome editing

CRISPR/Cas9

Baculovirus

Primary cells

\begin{abstract}
A B S T R A C T
Genome editing in eukaryotes became easier in the last years with the development of nucleases that induce double strand breaks in DNA at user-defined sites. CRISPR/Cas9-based genome editing is currently one of the most powerful strategies. In the easiest case, a nuclease (e.g. Cas9) and a target defining guide RNA (gRNA) are transferred into a target cell. Non-homologous end joining (NHEJ) repair of the DNA break following Cas9 cleavage can lead to inactivation of the target gene. Specific repair or insertion of DNA with Homology Directed Repair (HDR) needs the simultaneous delivery of a repair template. Recombinant Lentivirus or Adenovirus genomes have enough capacity for a nuclease coding sequence and the gRNA but are usually too small to also carry large targeting constructs. We recently showed that a baculovirus-based multigene expression system (MultiPrime) can be used for genome editing in primary cells since it possesses the necessary capacity to carry the nuclease and gRNA expression constructs and the HDR targeting sequences. Here we present new Acceptor plasmids for MultiPrime that allow simplified cloning of baculoviruses for genome editing and we show their functionality in primary cells with limited life span and induced pluripotent stem cells (iPS).
\end{abstract}

(C) 2017 Elsevier Inc. All rights reserved.

\section{Introduction}

Technologies for genome editing are important developments in molecular biology to study gene function. Genome editing allows modifications including insertion, deletion or point mutations to be made in a targeted gene (Hsu et al., 2014). Therefore, this technology holds much promise for addressing many fundamental as well as applied questions (Barrangou and Doudna, 2016) including, the generation of human disease models (Dow, 2015; Platt et al., 2014), ex vivo gene therapy (Benjamin et al., 2015; Savić and Schwank, 2016), and recombinant protein production (Peng et al., 2015). Four main classes of site specific DNA nucleases including meganuclease, zinc finger nuclease (ZFN), TALEN, and Cas9 have been developed to facilitate targeted genome editing in eukaryotic cells (Gaj et al., 2013; Nelson and Gersbach, 2016). The CRISPR/Cas9 system is currently the most popular tool due to its simplicity, high efficiency and versatility. Clustered regularly interspaced short palindromic repeats (CRISPRs), together with CRISPR-associated (Cas) proteins, provide adaptive immunity against viruses and plasmids in many bacterial species and most archaea (Jiang and Marraffini, 2015). The two crucial components in CRISPR/ Cas9-based genome editing are, Cas9 as a nuclease and a gRNA (Jinek et al., 2013; Ran et al., 2013). Together, Cas9 and the gRNA are able to

\footnotetext{
* Corresponding author at: Paul Scherrer Institute, Molecular Cell Biology, CH-5232 Villigen, Switzerland.

E-mail address: Philipp.Berger@psi.ch (P. Berger).
}

induce a double strand break (DSB) at specific sites in DNA of host cells. Subsequently, the DSB DNA damage is repaired by the cellular DNA repair machinery through either NHEJ or HDR pathways (Hsu et al., 2014; Jiang and Marraffini, 2015; Jinek et al., 2013). NHEJ is an error-prone process which fills the gaps with small insertions or deletions (indel) which can be taken advantage of to disrupt the coding region of a gene of interest (knockout). In contrast to NHEJ, the HDR pathway is suitable for precise genome editing by inserting (knockin) fragments of exogenous DNA at a precise position in the genome (Sander and Joung, 2014). However, HDR is an inefficient process that requires an exogenous repair DNA template with sequence homology to regions that flank the DSB (van Erp et al., 2015; Hsu et al., 2014; Sander and Joung, 2014; Wang et al., 2015). The recombinant viruses currently used for gene transfer, do not have sufficient capacity to carry Cas9 coding sequences, the gRNAs, and the construct for HDR in one vector.

One possibility for delivery of the necessary elements for CRISPR/ Cas9-HDR mediated knock-in is co-transfection or co-infection of Cas9, gRNA(s) and HDR constructs (Mali et al., 2013; Nelson and Gersbach, 2016). Co-transfection/co-infection is an easy and straightforward way to co-deliver multiple constructs, but it can cause low targeting efficiency in cells due to heterogeneity of vector delivery. In addition, some cells such as primary cells, are resistant to DNA transfection (Mansouri and Berger, 2014).

We recently showed that MultiPrime, a baculovirus-based system that was developed for multigene expression in primary cells, is suitable 
for genome editing because it has sufficient capacity to carry the CRISPR machinery and HDR targeting vectors (Mansouri et al., 2016). Here, we report new plasmids that allow simplified cloning of these constructs. Available elements from existing, commercial vectors like Cas9 or gRNAs are compatible with our cloning sites. As proof of concept, we tagged the HMGA1 locus with EGFP in HEK293 cells, an easy to transfect mammalian cell line. In addition, we transduced HUVEC and hiPS cells that are difficult to transfect. We demonstrate that our new plasmids support the rapid production of baculoviruses for genome editing in primary cells.

\section{Materials and methods}

\subsection{Vector design}

We designed an Acceptor plasmid for MultiPrime with unique cloning sites that allow the insertion of a nuclease, gRNAs, and a HDR construct, preferentially using fragments from CRISPR plasmids that are widely used in the genome engineering community (e.g. pX derivatives). The pSI-AG10 vector with additional I-SceI and I-CeuI sites was used as the backbone for the constructs. This part of the plasmid contains all of the necessary elements for MultiPrime baculovirus generation. pX derived vectors (including pX330, pX458, pX459, pX460, pX461, and pX462), contain a gRNA cassette composed of an U6 promoter-driven crRNA-tracrRNA that can be removed by AflIII/XbaI restriction digestion. We included two sites in our synthetic construct for insertion of two gRNAs ( $\mathrm{NcoI} \rightarrow \mathrm{XbaI}$ and $\mathrm{Bsal} \rightarrow$ NheI). Cas9 is flanked by Agel/NotI sites in the pX vectors. We have included the same restriction enzyme sites in our vector. Therefore, Cas9, or each variation of it including Cas9n or dCas9, can easily be placed at this position. The CMV promoter that drives Cas9 is localized between the Xbal/AgeI sites (in pSI-AG15/16 as well as in the pX plasmids). Thus, the CVM promoter sequences can be replaced by cell-type specific or regulatable elements for controlled expression of Cas9 if desired. In addition, an AflIII/ Notl fragment from the pX vector containing the gRNA and Cas9 expression cassette can be cloned directly into the NcoI/NotI digested pSIAG15/16 vector. The repair construct can be cloned into the Ascl/PacI sites in the vector. Alternatively, the repair construct can be cloned in parallel in a MultiPrime Donor vector and then inserted by a Cre/LoxPmediated recombination (Fig. 1a).

\subsection{Cloning of acceptor plasmids}

Plasmid pSI-AGZ10 was used as a template to construct the pSIAG15 vector (Mansouri et al., 2016). The AgeI site was removed from pSI-AG10 by digesting the plasmid with Agel followed by refilling with Phusion HF polymerase (NEB, USA) and relegation with T4 ligase (NEB, USA). The backbone of this plasmid was amplified using primers: AG15/16-forward-w-loxP 5'-CTA GGC TAG CGG TAT TAA CTA TAA CGG TCC TAA GGT AGC GAA ACC CAT CTA ATT GGA ACC AGA TAA G-3' and primer AG15/16-back-w-lox P 5'-CTT ACC ATG GGG ACC ATT ACC CTG TTA TCC CTA GTT ATA GTT ACA GAT AAC TTC-3' as forward and reverse

a

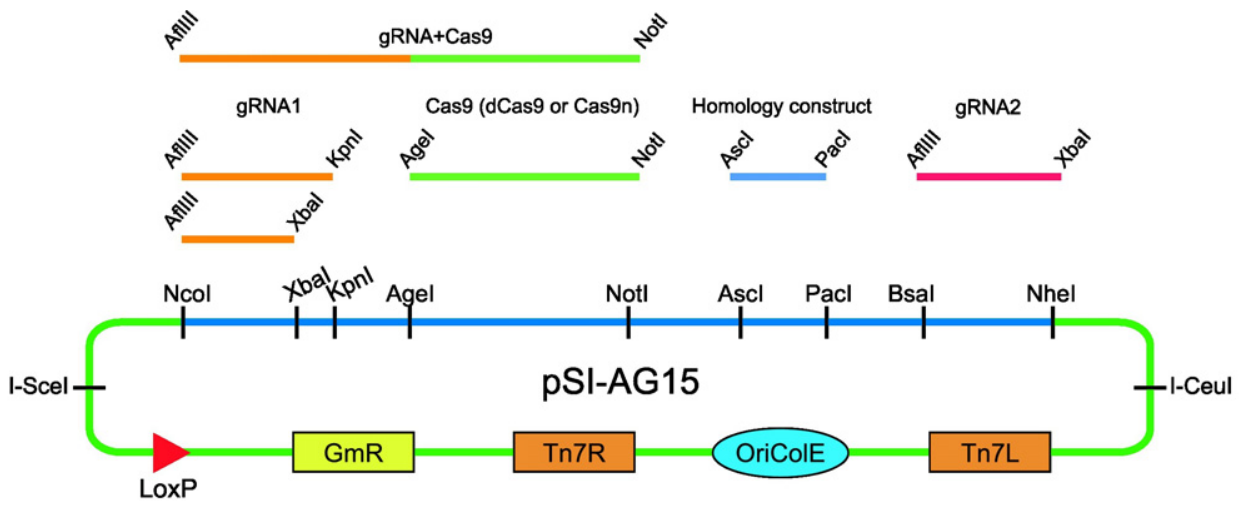

b

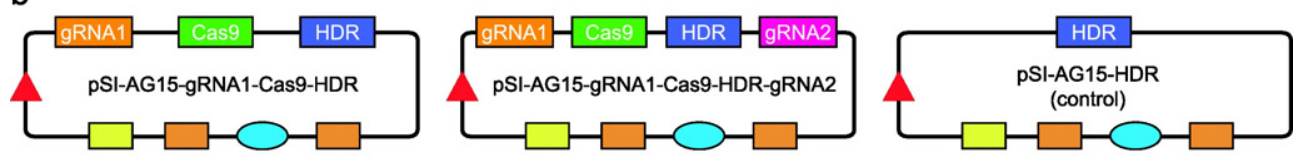

C

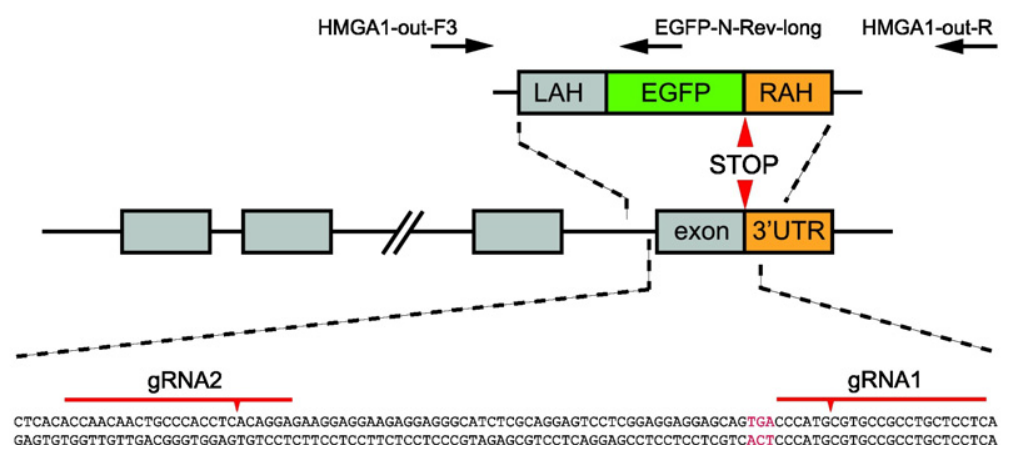

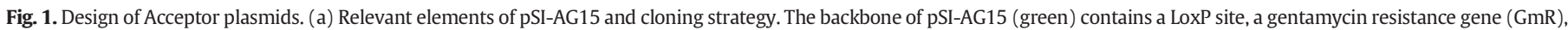

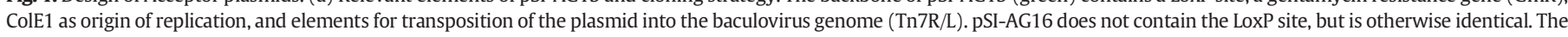

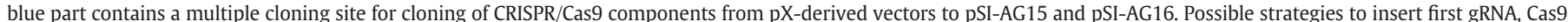

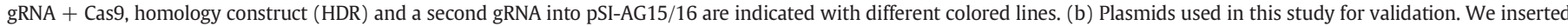

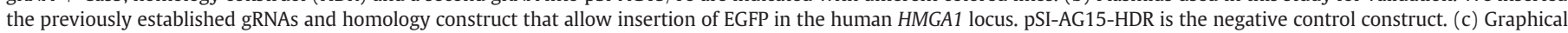
overview of the relevant part of the HMGA1 locus. Primers for genotyping are given on top, binding and cleavage sites of gRNAs are given at the bottom. 
primers, respectively. This resulted in a fragment with NcoI and NheI sites that was fused with a synthetic sequence (Genewiz, USA) containing unique restriction sites for the insert of all relevant elements for genome editing (Fig. 1a). In addition, we generated an Acceptor plasmid without LoxP site (pSI-AG16). The LoxP site was eliminated by site-directed mutagenesis using PCR with primer AG15/16-forward-wo-loxP 5'-CTA GGC TAG CGG TAT TAA CTA TAA CGG TCC TAA GGT AGC GAA ACC CAT CTA ATT GGA ACC AGA TAA G-3' and primer AG15/16-backwo-lox P5'-GGC GCC ATG GAC TTC GCA TTA CCC TGT TAT CCC TAT GCT GCC CAA GGT TGC CGG GTG AC-3' as forward and reverse primers, respectively.

\subsection{Cloning procedure}

Plasmids for targeting the HMGA1 locus were kindly provided by Dr. Stefan Jakobs (Ratz et al., 2015). Cas9 was excised together with gRNA1 by AflIII/NotI digestion from pX330-HMGA1-gRNA1 vector and cloned into the NcoI/NotI site of pSI-AG15 and pSI-AG16 generating the pSIAG15/16-gRNA1-Cas9 vector. The HDR construct AscI/Pacl fragment for insertion of EGFP was obtained from a pGEM-HDR vector and cloned AscI/PacI into pSI-AG15/16-gRNA1-Cas9 to generate pSI-AG15/16gRNA1-Cas9-HDR vector. The second HMGA1-gRNA (gRNA2) was isolated from pX330-HMGA1-gRNA2 as a AflIII/Xhol fragment and cloned into the BsaI/NheI digested pSI-AG15/16-gRNA1-Cas9-HDR vector to generate pSI-AG15/16-gRNA1-Cas9-HDR-gRNA2. Control plasmid pSIAG15/16-HDR was constructed by cloning of HDR through AscI/PacI to pSI-AG15/16 (Fig. 1b).

\subsection{Cell culture}

HEK293 cells were cultured in DMEM (Amimed) containing 10\% FBS (Life Technologies) and 100 units/ml Penicillin and $100 \mu \mathrm{g} / \mathrm{ml}$ Streptomycin (Life Technologies) in a humified incubator with $5 \% \mathrm{CO}_{2}$ at $37^{\circ} \mathrm{C}$. HUVECs (Life Technologies) were maintained in M-200 medium (Life Technologies). Human iPS cells (NAS2, kind gift from Tilo Kunath, University of Edinburgh) were cultured in mTESR1 medium (Stem Cell Technologies). Baculovirus generation in sf21 insect cells and transduction of mammalian cells was described previously (Mansouri et al., 2016). Transfection was performed with Lipofectamin 3000 (Life Technologies) according to manufacturer's recommendations. Cells were analyzed 5 or 6 days after transfection or transduction. The quantifications described in the result part are from single transduction or transfection experiment and represent mean \pm SEM from ten independent areas of three coverslips.

\subsection{Analysis of cells}

HEK293 and HUVECs were plated on glass slides coated with poly-Llysine (Sigma P4707) or 0.1\% gelatin (Sigma G1393). iPS cells were plated on hESC qualified Matrigel (BD Bioscience). Cells were fixed with $4 \%$ formaldehyde/PBS. iPS cells were immunostained with an Oct4 antibody (sc-5279, 1:500, Santa Cruz Technology) and nuclei were counter-stained with DAPI $(1 \mu \mathrm{g} / \mathrm{ml})$. Samples were mounted on glass slides with Gelvatol. Images were taken on a ZEISS Apotome.2 fluorescent microscope. Genomic DNA of cells was isolated by QiaAmp DNA mini Kit (Qiagen) and used as template in PCR genotyping reaction. PCR was performed in $1 \times\left(98^{\circ} \mathrm{C}\right.$ for $\left.5 \mathrm{~min}\right), 30 \times\left(98^{\circ} \mathrm{C}\right.$ for $30 \mathrm{~s}, 72^{\circ} \mathrm{C}$ for $30 \mathrm{~s}$ and $72{ }^{\circ} \mathrm{C}$ for $180 \mathrm{~s}$ ) and $1 \times\left(72{ }^{\circ} \mathrm{C}\right.$ for $\left.10 \mathrm{~min}\right)$. Primer HMGA1-out-F3 5'-TTG TCC TTC CTA TGA GCC TCT GCA G-3' was used as forward primer and either HMGA1-out-R 5'-CAA TGA CGG ATG TCG AAG AAT GGA ACA TTG AAC-3' or EGFP-N-Rev-long 5'-GTC AGC TTG CCG TAG GTG GCA TC-3' were used as reverse primers for amplification of wildtype or mutated alleles, respectively.

\section{Results}

\subsection{Designing of pSI-AG15 and pSI-AG16}

We showed previously that baculoviruses is an elegant tool for genome editing in mammalian cells and that it has sufficient capacity to harbor all relevant elements including a large homology construct (Mansouri et al., 2016). MultiPrime uses cre/LoxP technology to assemble multiple cassettes. This technology is extremely flexible but not essential for standardized applications. We therefore designed new acceptors for MultiPrime that allow straightforward cloning making use of commonly available CRISPR vectors (Fig. 1). To validate our vectors, we used constructs that were previously used to modify the human HMGA1 locus (Ratz et al., 2015). HMGA1 is a nuclear DNA-binding non-histone high mobility group protein. Targeting with these constructs leads to the expression of a HMGA1-EGFP fusion protein. Genome edited cells can easily be recognized by a green nucleus. The use of an established system allows us to compare our strategy with previously used strategies.

\subsection{Targeting of HMGA1 in HEK293 cells}

We first used HEK293 cells to compare the efficiencies of transfection and baculovirus transduction procedures. HEK293 cells are easy to transfect and we therefore do not expect a major difference in efficiency between the two methods. Image analysis by fluorescence microscopy revealed cells with green nuclei in transfected cells (data not shown) as well as in transduced cells (Fig. 2a). We observed approximately $4 \%$ edited cells following transfection which was similar to the efficiency observed by Ratz et al. for co-transfection of the targeting plasmids (Ratz et al., 2015). Transduction was more efficient and led to approximately $6 \%$ of targeted cells (Fig. 2b). Genotyping PCR and sequencing of the resulting PCR products confirmed correct integration of the HDR template. We only obtain a product for the mutated allele in cells that were transduced with a baculovirus that contains Cas9, gRNA, and repair construct (Fig. 2c). Sequence analysis of the mutant and the wildtype product show the correctly integrated and targeted sequence in the cell pool (Fig. 2d).

\subsection{Targeting of the HMGA1 gene in iPS cells and HUVECS}

A large number of cells and particularly primary cells are difficult to transfect with current reagents. We used iPS cells that are derived from primary human fibroblasts and primary HUVECs. iPS cells can go through clonal expansion whereas HUVEC have a limited life span and stop dividing after 7-10 passages. Again we compared transfection vs transduction for introducing the targeting construct in these two cell types. Only very few cells with green nuclei were observed in HUVEC and iPS cells following transfection with plasmids containing all three Cas9, gRNA and HDR targeting vector components (pSI-AG15_gRNA1Cas9-HDR or pSI-AG15_gRNA1-Cas9-HDR-gRNA2, data not shown). In contrast, transduction with baculoviruses containing the constructs led to more GFP-positive cells (Fig. 3a, d). Quantification revealed that approximately $4.5 \%$ (HUVEC) and 3\% (iPS) of the cells had been genome modified (11 and 4 times better than for transfection, respectively) (Fig. $3 b, e)$. Genotyping with PCR only resulted in a band for the mutated allele when all three components were transduced (Fig. 3c, f).

\section{Discussion}

A major challenge in the CRISPR/Cas9 system is safe and effective delivery of all components at the same time to host cells. This can be done by transfection or infection. We now developed Acceptor plasmids for the generation of baculovirus for genome editing. Baculovirus is superior to other viruses since it has enough capacity to harbor all elements that are necessary for genome editing with HDR. In addition, 

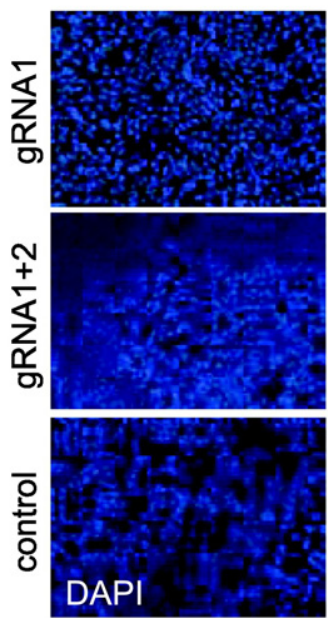

d

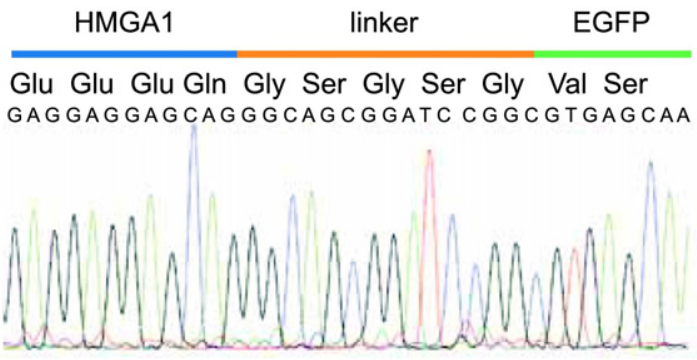

mutant

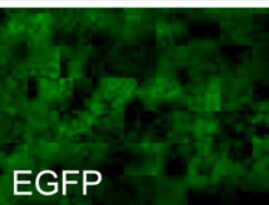

b

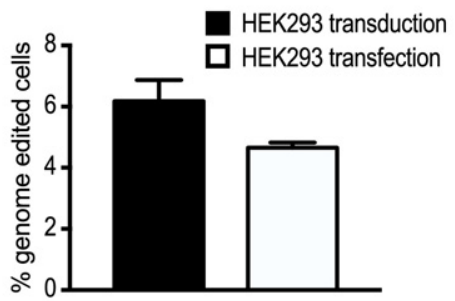

C
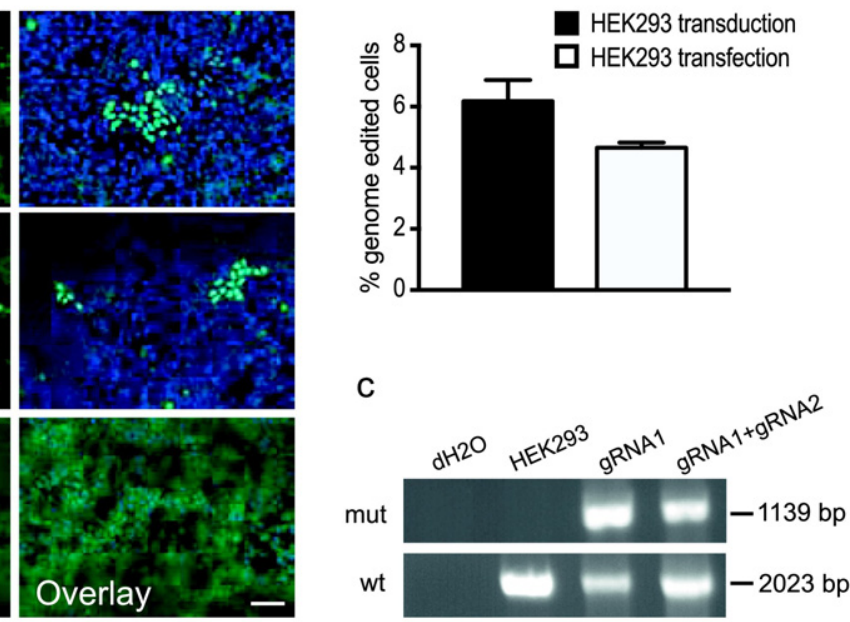

HMGA1

3' UTR

Glu Glu Gln Stop

AGGAGGAGCAGTGAC C CATGCGTGCCGC

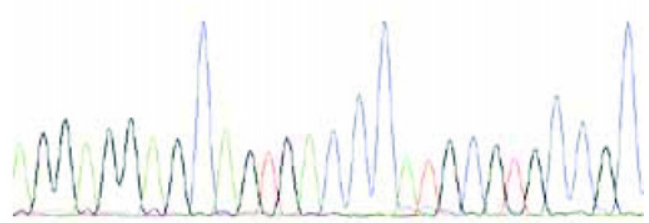

wildtype

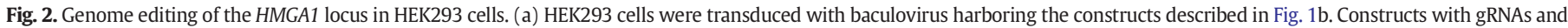

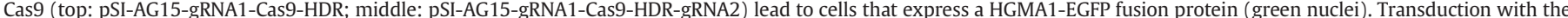

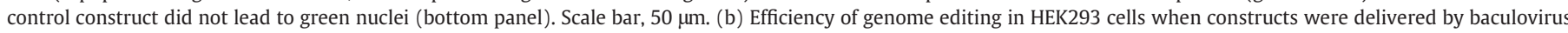

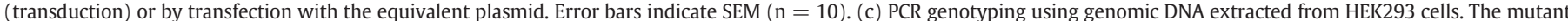

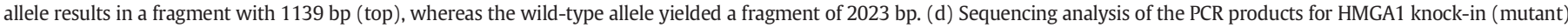
and wildtype locus.

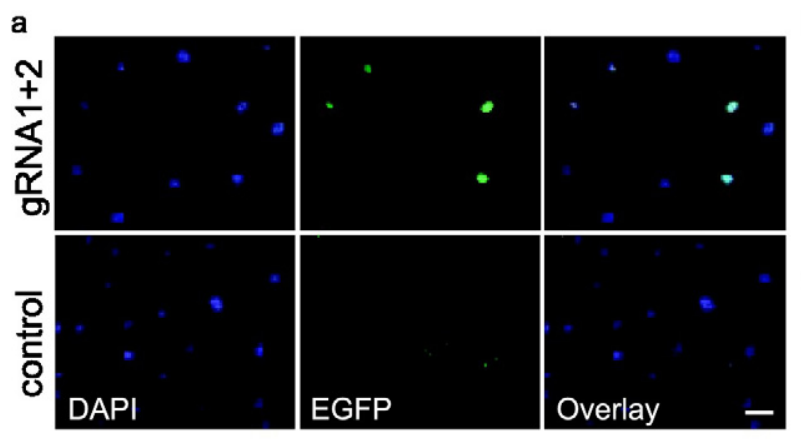

d

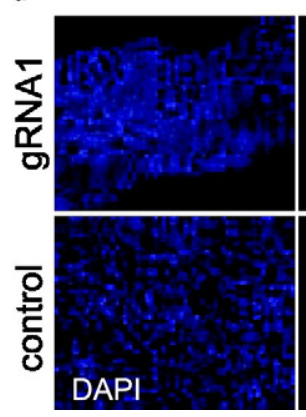

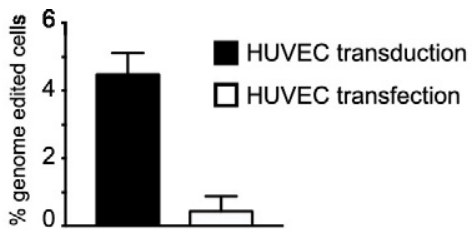
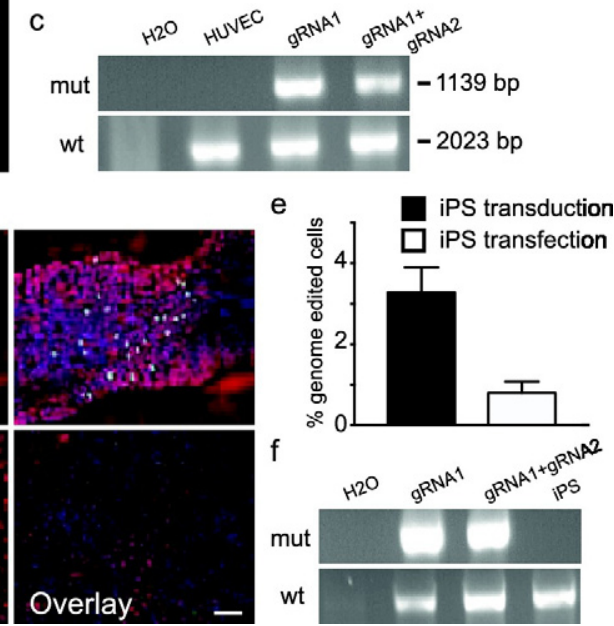

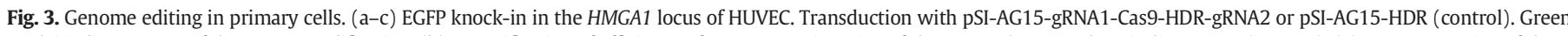

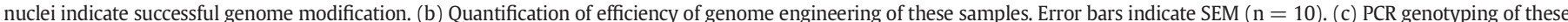

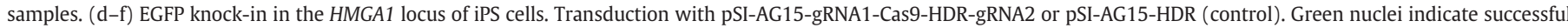

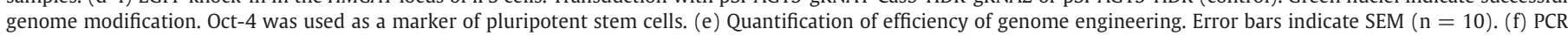
genotyping of these samples. Scale bar: $50 \mu \mathrm{m}$. 
baculovirus is a gentle tool to transfer DNA to cells. We showed before that it has little toxicity and the functionality of transduced cells is maintained (Mansouri et al., 2016). Adenovirus has 8-10 kb foreign DNA capacity and is therefore also a potential candidate for homology-based genome editing but it was to our knowledge not yet used for this purpose (Chen and Gonçalves, 2016; Kennedy and Parks, 2009; Luo et al., 2007).

Our results show that a simple transient transfection in easy-totransfect cells (e.g., HEK293) results in 4\% genome edited cells (Fig. 2). Transduction with a baculovirus led to better targeting but the increased efficiency may not be worth the investment to establish baculovirus-mediated targeting in these cell types. The situation changes in primary cells that are difficult to transfect. We observed very few genome targeted cells $(<1 \%)$ when we transfected HUVECs and iPS cells with our plasmids. This low efficiency is in many cases not sufficient to generate enough cells for analysis if targeted cells cannot be expanded extensively. In the case of iPS cells, clonal expansion to obtain $100 \%$ positive cell populations is possible but would require the parallel insertion of a selection marker which might be undesirable. HUVECs on the other hand undergo a limited number of doublings in vitro and divide only 5-7 times in culture. Clonal expansion is therefore not possible and the maximum possible modification rate must be reached with the targeted cells.

We never reached $>6 \%$ of modified cells in all cell types tested even though we routinely transduce $60-80 \%$ of all cells. This indicates that CRISPR mediated HDR is an inefficient process and that transfer of CRISPR elements does not guarantee successful modification. Future developments have to identify elements that enhance efficiency. MultiPrime has the advantage that it can also deliver these elements, e.g. proteins that enhance recombination efficiency or reduce off-target effects, as soon as they are identified. In addition, transient suppression of the NHEJ pathway using inhibitors of Ku70, Ku80 and DNA ligase IV might also improve HDR efficacy after transduction with baculovirus (Chu et al., 2015; Maruyama et al., 2015),

In summary, we constructed all-in-one plasmids encoding Cas9, gRNAs and HDR on a single plasmid. We increased efficiency of genome editing in primary cells and stem cells using baculovirus. We anticipate many applications in genome engineering (such as activation, repression, deletion or insertion of single or many genes) will benefit from our system.

\section{Acknowledgement}

This work was supported by the Swiss National Science Foundation (31003A_146975 to PB). We would like to thank Dr. Stefan Jakobs for plasmids. This work was also supported by the Swiss National Science Foundation through SystemX.ch under the RTD project NeuroStemX to VT.

\section{References}

Barrangou, R., Doudna, J.A., 2016. Applications of CRISPR technologies in research and beyond. Nat. Biotechnol. 34, 933-941.

Benjamin, D., Cox, T., Platt, R.J., Zhang, F., 2015. Therapeutic genome editing: prospects and challenges. Nat. Med. 21.

Chen, X., Gonçalves, M.A.F.V., 2016. Engineered viruses as genome editing devices. Mol. Ther. 24, 447-457.

Chu, V.T., Weber, T., Wefers, B., Wurst, W., Sander, S., Rajewsky, K., Kühn, R., 2015. Increasing the efficiency of homology-directed repair for CRISPR-Cas9-induced precise gene editing in mammalian cells. Nat. Biotechnol. 33, 543-548.

Dow, L.E., 2015. Modeling disease in vivo with CRISPR/Cas9. Trends Mol. Med. 21, 609-621.

van Erp, P.B., Bloomer, G., Wilkinson, R., Wiedenheft, B., 2015. The History and Market Impact of CRISPR RNA-Guided Nucleases.

Gaj, T., Gersbach, C.a., Barbas, C.F., 2013. ZFN, TALEN, and CRISPR/Cas-based methods for genome engineering. Trends Biotechnol. 31, 397-405.

Hsu, P.D., Lander, E.S., Zhang, F., Barrangou, R., van der Oost, J., Barrangou, R., Fremaux, C., Deveau, H., Richards, M., Boyaval, P., et al., 2014. Development and applications of CRISPR-Cas9 for genome engineering. Cell 157, 1262-1278.

Jiang, W., Marraffini, L.A., 2015. CRISPR-Cas: new tools for genetic manipulations from bacterial immunity systems. Annu. Rev. Microbiol. 69, 209-228.

Jinek, M., East, A., Cheng, A., Lin, S., Ma, E., Doudna, J., 2013. RNA-programmed genome editing in human cells. eLife 2, e00471.

Kennedy, M.A., Parks, R.J., 2009. Adenovirus virion stability and the viral genome: size matters. Mol. Ther. 17, 1664-1666.

Luo, J., Deng, Z.-L., Luo, X., Tang, N., Song, W.-X., Chen, J., Sharff, K.A., Luu, H.H., Haydon, R.C., Kinzler, K.W., et al., 2007. A protocol for rapid generation of recombinant adenoviruses using the AdEasy system. Nat. Protoc. 2, 1236-1247.

Mali, P., Yang, L., Esvelt, K.M., Aach, J., Guell, M., DiCarlo, J.E., Norville, J.E., Church, G.M., 2013. RNA-guided human genome engineering via Cas9. Science 339, 823-826.

Mansouri, M., Berger, P., 2014. Strategies for multigene expression in eukaryotic cells. Plasmid 75, 12-17.

Mansouri, M., Bellon-Echeverria, I., Rizk, A., Ehsaei, Z., Cianciolo Cosentino, C., Silva, C.S., Xie, Y., Boyce, F.M., Davis, M.W., Neuhauss, S.C.F., et al., 2016. Highly efficient baculovirus-mediated multigene delivery in primary cells. Nat. Commun. 7, 11529.

Maruyama, T., Dougan, S.K., Truttmann, M.C., Bilate, A.M., Ingram, J.R., Ploegh, H.L., 2015. Increasing the efficiency of precise genome editing with CRISPR-Cas9 by inhibition of nonhomologous end joining. Nat. Biotechnol. 33, 538-542.

Nelson, C.E., Gersbach, C.A., 2016. Engineering delivery vehicles for genome editing. Annu. Rev. Chem. Biomol. Eng. 7, 637-662.

Peng, J., Wang, Y., Jiang, J., Zhou, X., Song, L., Wang, L., Ding, C., Qin, J., Liu, L., Wang, W., et al., 2015. Production of human albumin in pigs through CRISPR/Cas9-mediated knockin of human cDNA into swine albumin locus in the zygotes. Sci. Rep. 5, 16705.

Platt, R.J., Chen, S., Zhou, Y., Yim, M.J., Swiech, L., Kempton, H.R., Dahlman, J.E., Parnas, O. Eisenhaure, T.M., Jovanovic, M., et al., 2014. CRISPR-Cas9 knockin mice for genome editing and cancer modeling. Cell 159, 440-455.

Ran, F.A., Hsu, P.D., Wright, J., Agarwala, V., Scott, D.A., Zhang, F., 2013. Genome engineering using the CRISPR-Cas9 system. Nat. Protoc. 8, 2281-2308.

Ratz, M., Testa, I., Hell, S.W., Jakobs, S., 2015. CRISPR/Cas9-mediated endogenous protein tagging for RESOLFT super-resolution microscopy of living human cells. Sci. Rep. 5, 9592.

Sander, J.D., Joung, J.K., 2014. CRISPR-Cas systems for editing, regulating and targeting genomes. Nat. Biotechnol. 32, 347-355.

Savić, N., Schwank, G., 2016. Advances in therapeutic CRISPR/Cas9 genome editing. Transl. Res. 168, 15-21.

Wang, B., Li, K., Wang, A., Reiser, M., Saunders, T., Lockey, R.F., Wang, J.W., 2015. Highly efficient CRISPR/HDR-mediated knock-in for mouse embryonic stem cells and zygotes. Biotechniques 59 (4), 201-202, 204, 206-208. 